C2008 IEEE. Personal use of this material is permitted. However, permission to reprint/republish this material for advertising or promotional purposes or for creating new collective works for resale or redistribution to servers or lists, or to reuse any copyrighted component of this work in other works must be obtained from the IEEE. 


\section{State of the Art in Negotiation Ontologies for Enhancing Business Intelligence}

\author{
Hai Dong \\ Curtin University of \\ Technology \\ hai.dong@cbs.curtin.edu.au
}

\author{
Farookh Khadeer Hussain \\ Curtin University of \\ Technology \\ hussain.farookh@cbs.curti \\ n.edu.au
}

\author{
Elizabeth Chang \\ Curtin University of \\ Technology \\ elizabeth.chang@cbs.curti \\ n.edu.au
}

\begin{abstract}
Negotiation is defined as two or more agents making agreement on the common interests, which provides an efficient approach for companies to gain business intelligence from suppliers and customers. Current negotiation research focuses on automatically stimulating negotiation behaviors among agents. Ontology, as a modeling technology in semantic web, is regarded as an effective tool to improve the negotiation ability of agents, considering that ontology can provide richer semantic expressions for agents in negotiation process. In this paper, we make a brief review on the current negotiation ontology researches. In addition, by means of a unique ontology notation system, we unify the notations used in these ontologies, to maximally promote knowledge sharing outcome in this field. By comparing and contrasting the ontologies from five perspectives, we draw a brief conclusion to the present status of negotiation ontology researches, and make our suggestions to the further research in this field.
\end{abstract}

\section{Introduction}

Negotiation is defined as two or more agents making agreement on the common interests [2] [13]. In the last few years, negotiation research has gained increasing attentions from the scholars with the background of business and computer science. Agent and ontology technology are utilized to generate automatic negotiation process. In this paper, we will make a brief review on the researches regarding negotiation ontologies.

Ontology is defined as "a specific artifact designed with the purpose of expressing the intended meaning of a shared vocabulary", "a shared vocabulary plus a specification of its intended meaning" or " $a$ specification of a conceptualization" [5]. The benefit of ontology is that by means of organizing knowledge in specific domains, ontology may be utilized to promote knowledge sharing within organizations or inter-organizations [4]. Ontology can benefit the negotiation behavior among agents by sharing negotiation knowledge. However, in current negotiation research, one issue is that various notation systems are used to represent ontology, such as SO1, AMW2, UML3 and so forth. This could negatively affect the realization of knowledge sharing in the negotiation research field. Thus, another task in this paper is to unify the ontology notations, by introducing a simple ontology notation system. In addition, we briefly compare these ontologies from five perspectives. The conclusion to this survey is drawn in the final section.

\section{Business intelligence and negotiation}

Business intelligence (BI) refers to using various applications, technologies and practices to improve decision-making performance, by means of collecting, integrating and analyzing business information [15]. The objects of gaining BI generally can be divided into two main aspects - internal and external. The internal object refers to daily operations. The external object refers to customers, competitors, business partners and economic environment [16]. Negotiation is one of the methods which help companies to gain BI from customers and business partners, by building customer or business partner relationships, learning customer or business partner behaviors, gaining customer or business partner suggestions and so forth [17]. Thus, 
negotiation can provide an efficient approach for gaining $\mathrm{BI}$.

\section{Ontology notation system for unifying negotiation ontology expressions}

The notation system utilized in the ontological representation is based on Chang et al.'s work, which consists of four basic notations as Table 1[3]. Although in most researchers use UML to represent ontology model, due to its complex symbol system, UML cannot efficiently help people without software engineering knowledge to better understand the shared knowledge. This notation system simplifies the symbols and its symbols are closer to the principle of ontology which is the combination of shared concepts and relationships between concepts [5].

\section{Negotiation ontology researches}

The current negotiation researches focus on negotiation protocols and negotiation strategies. The former mainly refers to generating rules and regulations for new participants to adapt to negotiation environments; the latter refers to the principle about selecting different actions to respond in different negotiation scenarios [13]. Most negotiation ontologies are designed to automatically generate protocols for the negotiation behaviour in e-business context. The
Table 1. Ontological notation system

\begin{tabular}{|l|l|}
\hline $\begin{array}{l}\text { Ontology } \\
\text { Notations }\end{array}$ & Semantics of the Notations \\
\hline & $\begin{array}{l}\text { Double-field Box represents the } \\
\text { Ontological Concepts. }\end{array}$ \\
\hline- relation $_{-}-$ & $\begin{array}{l}\text { A dotted line represents Ontology } \\
\text { Concept Association Relation } \\
\text { which represents a Concept is } \\
\text { closely related to another concept. } \\
\text { The relationship name can be } \\
\text { noted above the dotted line. }\end{array}$ \\
\hline$\rightarrow$ & $\begin{array}{l}\text { Open-arrow line represents } \\
\text { Composition and Aggregation or } \\
\text { Part-of relationship between Upper } \\
\text { Ontology Concept and Lower } \\
\text { Ontology Concept. }\end{array}$ \\
\hline $\begin{array}{l}\text { Solid-arrow line represents } \\
\text { Generalization and Specification } \\
\text { relation, which is a relation } \\
\text { between Upper Generic Concept } \\
\text { and Lower Specific Concept. }\end{array}$ \\
\hline $\begin{array}{l}\text { Circle-line represents the instance } \\
\text { of Ontological Concepts. }\end{array}$ \\
\hline$\longrightarrow$
\end{tabular}

following negotiation ontologies will be delivered

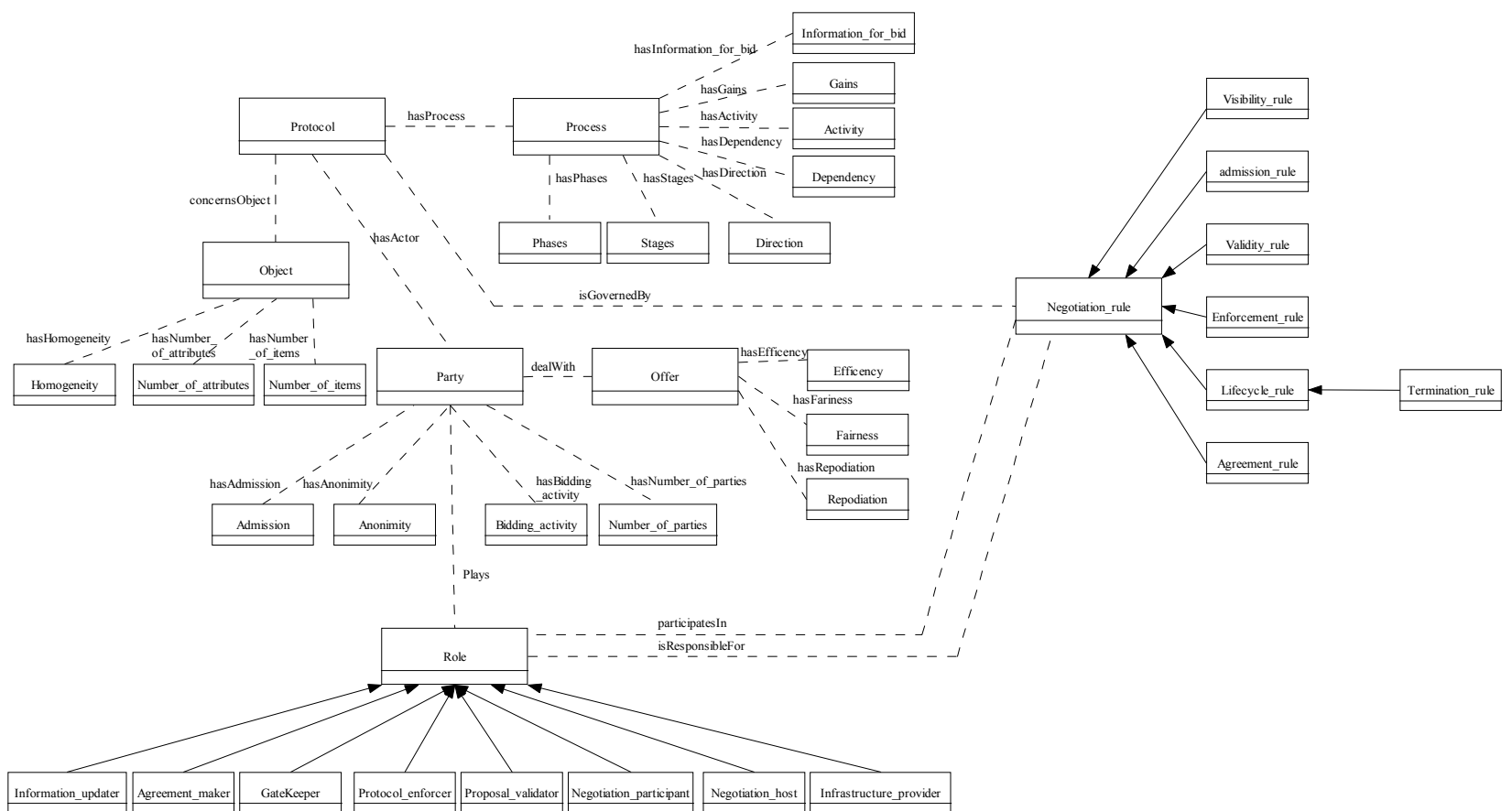

Figure 1. Negotiation ontology for e-commerce 
based on the different functions or working environment.

\subsection{Ontology-based negotiation protocols}

As described before, many researchers have concentrated on creating negotiation protocols by means of ontology.

Tamma et al. created a negotiation protocol ontology in the context of e-commerce [13] [14]. Based on London classification which identifies the concepts in most negotiation protocols, Tamma's ontology can be mainly divided into three parts (Fig. 1). The first part is "people", which involves the participants and their roles in negotiation processes. The second part is "goods", which refers to the objects of negotiation. The final part is "process", which contains the steps and attributes to reach an agreement. In addition, the protocol is affected by negotiation rules set up for different functions.

Strobel proposed a negotiation ontology in the context of e-market [11]. In the future, negotiation processes occur between software agents instead of humans. The research focused on designing negotiation scenario. Based on a series of literature review, the author developed the steps of an e-transaction advertisement, bid and contract. Negotiation exists between the 2nd and 3rd step. The negotiation ontology is presented from the perspective of behaviour and structure (Fig. 2). Then Strobel provided the communication design which distinguishes offerto-buy and offer-to-sale, and designs the structure of offer state. Finally, he implemented the ontology by means of XML.

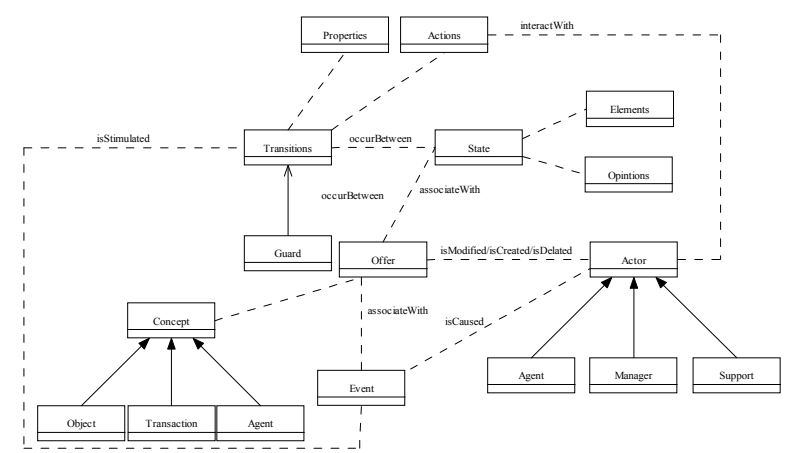

Figure 2. Negotiation ontology for e-market

\subsection{Negotiation ontologies for solving heterogeneity problems}

In addition, some researchers attempt to solve the heterogeneous issues existing in the negotiation process, in term of ontology engineering.
Bravo et al. focused on the incompatibility of protocols installed in agents with different negotiating ontologies, thus design a new ontology involving a shared vocabulary of negotiation terms and messages as well as a semantic disambiguation module, to release the misunderstandings between agents with different protocol ontologies [2]. First of all, Bravo studied the execution sequence of transaction in the context of e-market, then presented the architecture of negotiation execution processes with three main layers - publication layer, negotiation layer and semantic layer. Finally, the researchers presented the negotiation protocol and the negotiation ontology (Fig. 3).

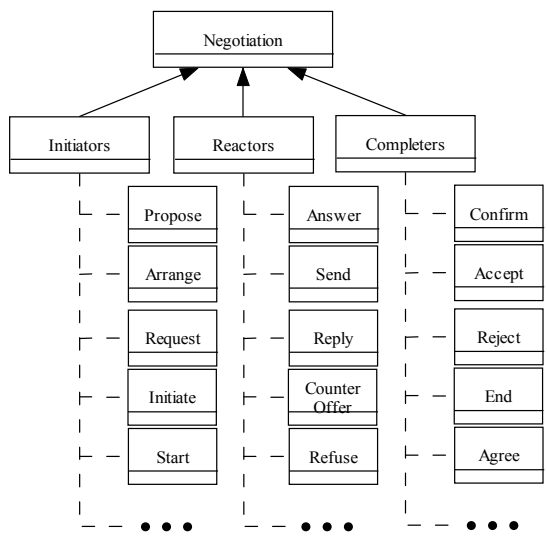

Figure 3. General structure of negotiation ontology

Malucelli et al. recognized the semantic heterogeneity issues in negotiation process [8]. In the traditional negotiation ontologies, when a new agent wants to participate in a negotiation process, it has to be reprogrammed to suit to the protocol existing in the

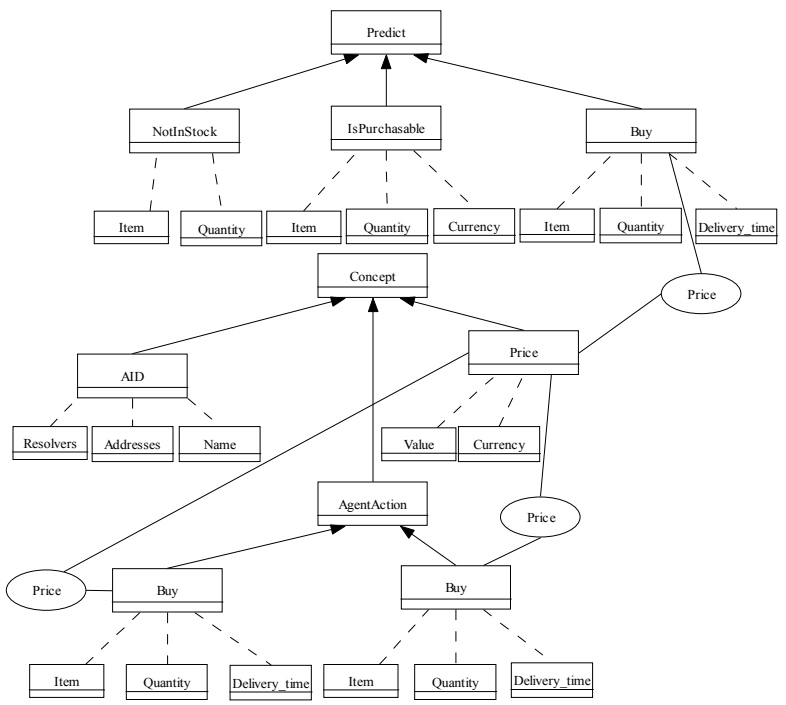

Figure 4. E-commerce ontology 
process. To challenge the issue, Malucelli utilized the JADE (Java Agent Development Framework) platform and OWL (Web Ontology Language) to construct a two-double ontological architecture. The top-level ontology namely E-commerce ontology (Fig. 4) contains shared negotiation terms and messages for all agents. For the specific negotiation, the agent is coded with the domain-specific ontology. Thus, this design can be seen as a solution for the semantic heterogeneity problem in negotiation process.

\subsection{SEWASIE}

SEWASIE (Semantic Webs and Agents in Integrated Economics) is a project in which the researchers utilize ontology to support the negotiation service among the SMEs [10].

Becks et al. described the use of negotiation ontology as the basis of business contract [1]. In SWEASIE, the negotiation process can be divided into three phases - business partner searching phase, negotiation process phase and fulfillment phase. By designing negotiation ontology, the terms searched in the first phase can be analyzed and shared in the second phase, and merged ontologies in the second phase can be extended as users' need. Becks also introduced the work flow of the ontology support in negotiation process. After a specific-domain ontology is searched in the first phase, the ontology can be refined and extended according to users' requirements, then be formed a negotiation ontology with a default contract ontology, which can be used as the basis for contract negotiation and contract format.

\subsection{Meaning negotiation}

Moor discussed the role of negotiation ontology in communities of practice (CoP) [9]. In CoP, communities have strong need of communication, in the purpose of coordinating internal actions. However, facilitating proper service is difficult, since technologybased methods cannot satisfy the communicative requirement. The reason is that the communities are managed by communicative norms, which needs modeling interaction patterns within communities. Here, the key point is that the terms in the models need to be agreed by negotiation in communities. Thus, the researcher focused on developing ontologies for meaning negotiation processes. DOGMA is a framework that focuses on achieving agreement on meaning among communities, by means of an ontology server. Based on the DOGMA, Moor contributed to the ontology guided meaning processes.

\subsection{Negotiation and corporate learning}

Supnithi et al. focused on negotiation process in collaborative learning [12]. The negotiation process is concerned with sharing learning goals in the learning process. Then the authors constructed an "opportunistic group formation" model for the negotiation process. Two ontologies are contained in this model, which are negotiation ontology (Fig. 5) and collaborative learning ontology. The essence of reaching an agreement in a learning group is to share a negotiation ontology among agents who represent group members. By sharing the ontology, each agent can understand others' learning goals and thus are able to negotiate. Collaborative learning ontology is to define the collaborative process.

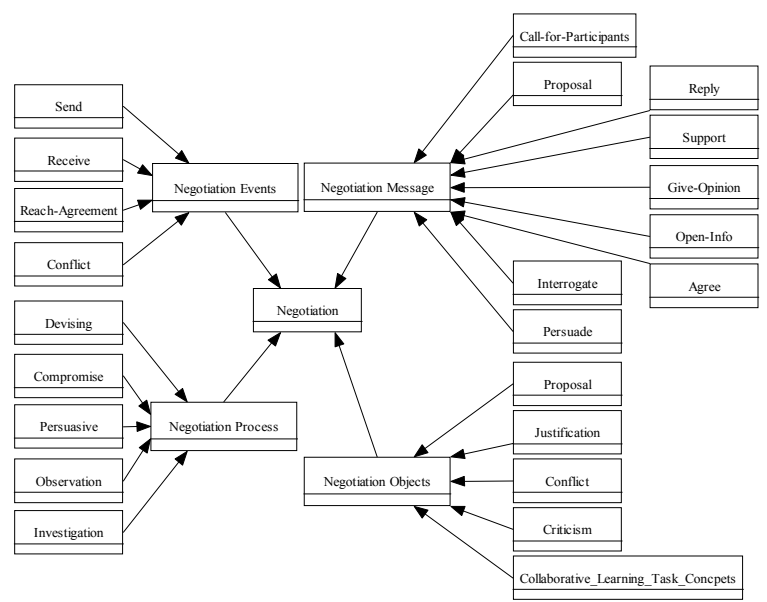

Figure 5. Hierarchy of negotiation ontology

\subsection{Trust Negotiation}

Bertino et al. described the concept of Trust Negotiation (TN), the elements of TN Systems, and defined the language and system requirement for TN. $\mathrm{TN}$ is defined as "a promising approach for establishing trust in open systems such as the Internet, where sensitive interactions sometimes occur among

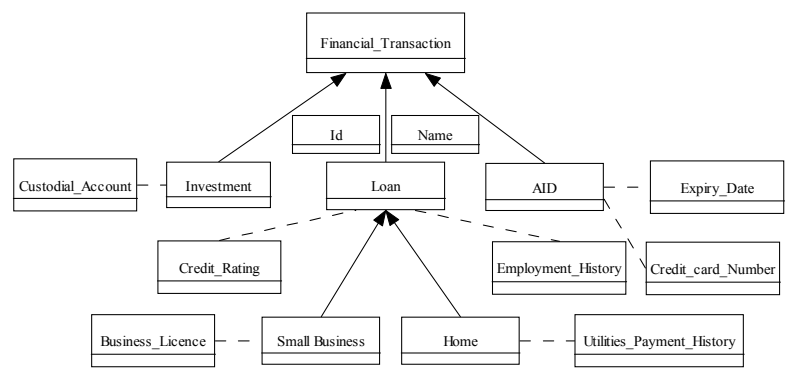

Figure 6. Negotiation ontology for a typical financial transaction 
entities with no prior knowledge of each other". Trust is built by "iteratively disclosing digital credentials according to ad hoc resources-namely, disclosure policies" [7]. When a party wishes to access a resource owned by another party, $\mathrm{TN}$ is triggered. The purpose of $\mathrm{TN}$ is to share information about credentials between both negotiation parties. In negotiation process, it is recognised that unnecessary questions should be avoided, in order to protect sensitive information. Ontology can be used as an efficient methodology, in order to define the attributes of negotiation content. Here the authors develop a negotiation ontology for a typical financial transaction (Fig. 6) [18].

\section{Compare and contrast of the existing negotiation ontologies}

In this section, we briefly compare and contrast these negotiation ontologies from the perspective of domain, functions, implementation techniques, evaluation method and result (Table 2 ).

From Table 2, it is observed that most (5 of 8) of the negotiation ontologies are designed for the ebusiness field, which indicates the urgent requirement of negotiation techniques in this field. The purpose of ontology design mostly focuses on solving understanding ambiguous problems among two or more negotiation parties (agents) within various interactive activities, such as business negotiation, collaborative learning, community information sharing and so forth. The implementation of ontologies is based on Protégé incorporated with diverse Java-based platforms, e.g. JADE (Java Agent Development Framework). Since only a few (2 of 8 ) cases provide their evaluation details and results, we cannot deduce the overall performance of these negotiation ontologies.

Thus, it can be concluded that the research of negotiation ontology is still in infancy, which has not completely revealed the power of ontology in negotiation processes. It is suggested that apart from providing conceptual models, the authors are also needed to provide more implementation and evaluation details, to prove the effectiveness and efficiency of their negotiation ontologies.

\section{Conclusion}

In this paper, we make a brief review in the field of

Table 2. Compare and contrast of the negotiation ontologies

\begin{tabular}{|c|c|c|c|c|c|}
\hline Name & Domain & Functions & $\begin{array}{l}\text { Implementation } \\
\text { Techs }\end{array}$ & $\begin{array}{l}\text { Evaluation } \\
\text { Method }\end{array}$ & $\begin{array}{l}\text { Evaluation } \\
\text { Result }\end{array}$ \\
\hline \begin{tabular}{|l|}
$\begin{array}{l}\text { Negotiation } \\
\text { ontology } \\
\text { [13] [14] }\end{array}$ \\
\end{tabular} & E-commerce & $\begin{array}{l}\text { Ontology-based } \\
\text { negotiation protocol. }\end{array}$ & Protege & $\begin{array}{l}\text { Being tested in a JASA } \\
\text { auction simulator. }\end{array}$ & Not provided. \\
\hline $\begin{array}{l}\text { Negotiation } \\
\text { Ontology } \\
\text { [11] }\end{array}$ & E-market & $\begin{array}{l}\text { Negotiation support } \\
\text { for bid and contract } \\
\text { in e-transaction. }\end{array}$ & $\mathrm{XML}$ & $\begin{array}{l}\text { Being tested in an } \\
\text { enScenario environment - } \\
\text { an electronic negotiation } \\
\text { scenario. }\end{array}$ & $\begin{array}{l}\text { The representation } \\
\text { is not complete } \\
\text { for unstructured } \\
\text { communications. }\end{array}$ \\
\hline $\begin{array}{l}\text { Negotiation } \\
\text { Ontology } \\
{[2]}\end{array}$ & Generic & $\begin{array}{l}\text { Ontology support for } \\
\text { solving semantic } \\
\text { heterogeneity issues } \\
\text { among agents in } \\
\text { negotiation process. }\end{array}$ & $\begin{array}{l}\text { J2SE, UDDI4J, } \\
\text { JAXR, JDBC APIs, } \\
\text { and BPEL4WS. }\end{array}$ & $\begin{array}{l}\text { Comparing the negotiation } \\
\text { performance before and } \\
\text { after the negotiation } \\
\text { ontology is utilized. }\end{array}$ & $\begin{array}{l}\text { A deduction of } \\
\text { no-understanding } \\
\text { in negotiation. }\end{array}$ \\
\hline $\begin{array}{l}\text { E-commerce } \\
\text { Ontology [8] }\end{array}$ & E-commerce & $\begin{array}{l}\text { Ontology support for } \\
\text { solving heterogeneity } \\
\text { issues in e-commerce } \\
\text { B2B negotiations. } \\
\end{array}$ & $\begin{array}{l}\text { Protege and } \\
\text { JADE. }\end{array}$ & Not provided. & Not provided. \\
\hline SEWASIE [1] & E-business & $\begin{array}{l}\text { Ontology support for } \\
\text { negotiaions and } \\
\text { contracts. } \\
\end{array}$ & JADE & Not provided. & Not provided. \\
\hline $\begin{array}{l}\text { Meaning } \\
\text { Negotiation } \\
{[9]}\end{array}$ & Communities & $\begin{array}{l}\text { Ontology support for } \\
\text { meaning negotiations } \\
\text { within communications } \\
\text { among communities. } \\
\end{array}$ & Not provided & Not provided. & Not provided. \\
\hline \begin{tabular}{|l|} 
Negotiation \\
Ontology [12]
\end{tabular} & $\begin{array}{l}\text { Collaborative } \\
\text { learning }\end{array}$ & $\begin{array}{l}\text { Ontology support for } \\
\text { sharing learning goals. }\end{array}$ & Not provided & Not provided. & Not provided. \\
\hline \begin{tabular}{|l|} 
Trust \\
Negotiation \\
Ontology [18]
\end{tabular} & Generic & $\begin{array}{l}\text { Sharing information } \\
\text { regarding credentials of } \\
\text { each negotiation parties. }\end{array}$ & Not provided & Not provided. & Not provided. \\
\hline
\end{tabular}


negotiation ontologies, which is an emerging research field focusing on stimulating automatic negotiation behaviours among agents by means of semantic web technologies. In addition, one issue observed by us in this field, is that the surveyed negotiation ontologies utilize personalized ontological annotation systems. This phenomenon could negatively affect knowledge sharing outcome in this field. Thus, apart from making an overall review on these researches, we present a set of simple ontological annotations, to unify the annotations used in the ontologies. We review the researches from six categories based on their distinctive functions and working environments. Finally, we compare and contrast them from five perspectives and draw our conclusions toward this survey.

\section{References}

[1] A. Becks, J. Huster, M. Jarke, A. Jertila, D. Kensche, C. Quix, and C. Seeling, "Value-Added Services enabling Semantic Web Technologies for SMEs," in the 4th International Semantic Web Conference, Galway, 2005.

[2] M. C. Bravo, J. Perez, V. J. Sosa, A. Montes, and G. Reyes, "Ontology Support for Communicating Agents in Negotiation Processes," in the Fifth International Conference on Hybrid Intelligent Systems, Rio de Janeiro, 2005, pp. 482487.

[3] E. Chang, T. S. Dillon, and F. Hussain, Trust and Reputation for Service Oriented Environments-Technologies for Building Business Intelligence and Consumer Confidence: John Wiley \& Sons, 2005.

[4] A. Gómez-Pérez and O. Corcho, "Ontology specification languages for the semantic web," IEEE Intelligent Systems, vol. 17, pp. 54-60, 2002.

[5] N. Guarino, "Ontology-Driven Conceptual Modelling," Trento-Roma: Institute for Cognitive Science and Technologies, 2003.

[6] R. Herrler, C. Heine, and F. Klügl, "Appointment Scheduling Among Agents: a case study in designing suitable interaction protocols," in Eighth Americas Conference on Information Systems, Dallas, 2002, pp. 14561463.
[7] E. Bertino, E. Ferrari, and A. Squicciarini, "Trust negotiations: concepts, systems and languages," Web Engineering, vol. July/August, 2004.

[8] A. Malucelli, D. Palzer, and E. n. Oliveira, "Ontologybased services to help solving the heterogeneity problem in e-commerce negotiations," Electronic Commerce Research and Applications, vol. 5, pp. 29-43, 4 October 20062006.

[9] A. d. Moor, "Ontology-guided meaning negotiation in communities of practice," in 2nd International Conference on Communities and Technologies, Milano, 2005.

[10] M. Schoop, M. U. Rehman, and A. Jertila, "Specification of agent technology for negotiation support," Informatik V, Aachen 2004.

[11] M. Strobel, "An XML schema representation for the communication design of electronic negotiations," Computer Networks, vol. 39, pp. 661-680, 2002.

[12] T. Supnithi, A. Inaba, M. Ikeda, J. i. Toyoda, and R. Mizoguchi, "Learning goal ontology supported by learning theories for opportunistic group formation " in AIED' 99, 1999.

[13] V. Tamma, S. Phelps, I. Dickinson, and M. Wooldridge, "Ontologies for supporting negotiation in e-commerce," Engineering Applications of Artificial Intelligence, vol. 18, pp. 223-236, 2005.

[14] V. Tamma, M. Wooldridge, and I. Dickinson, "An ontology for automated negotiation," in AMEC 02, Bologna, 2002, pp. 219-237.

[15] D. J. Power, "A brief history of decision support systems," 2.8 ed: DSSResources.COM, 2003.

[16] "What is business intelligence," Tech-FAQ, 2008.

[17] E. Chang, F. K. Hussain, and T. Dillon, "CCCI metrics for the measurement of quality of e-service," in the 2005 IEEE/WIC/ACM International Conference on Intelligent Agent Technology (IAT' 05), Compiegne University of Technology, 2005, pp. 603-610.

[18] T. Leithead, W. Nejdl, D. Olmedilla, K. E. Seamons, M. Winslett, T. Yu, and C. C. Zhang, "How to exploit ontologies in trust negotiation," in the 3rd ISWC Workshop on Trust, Security, and Reputation on the Semantic Web, Hiroshima, 2004. 